\section{RECLUST-A FORTRAN program for cluster analysis of multitrial free recall data}

\author{
CHARLES E. LINCOLN \\ Rice University, Houston, Texas 77001
}

The measurement of subjective organization using data from free recall paradigms has been widely utilized in semantic memory research beginning with the studies by Bousfield (1953) and Bousfield and Sedgewick (1944), which first demonstrated the phenomenon of output clustering based upon semantic similarity. Since then, a number of measurement techniques have been devised in efforts to quantify different aspects of this organizational structure. Most of these methods (see Sternberg \& Tulving, 1977, for a review) provide only a unidimensional index of the amount of organization, with little or no information about the structure of the subject's internal organization. Friendly (1977, Note 1) and Miller (1969) have suggested techniques for using measures of interitem proximities for analysis of subjective organizational structures. These proximities can be produced either by a categorical sorting procedure or from recall adjacency measures such as interresponse times. The resulting proximity matrix is then cluster analyzed to identify subjective organizational units. RECLUST is designed specifically to analyze free recall data, although it will accept as input a proximity matrix produced by any scaling procedure.

Algorithm. The raw distance, $\mathrm{D}_{\mathrm{ij}}$, between word $\mathrm{i}$ and word $j$ is defined as follows:

$$
D_{i j}=\frac{\sum_{n=1}^{N} \phi_{i j n}\left(\underset{k=i}{\sum} d_{k}\right)}{\sum_{n=1}^{N} \phi_{i j n}},
$$

where $\mathbf{N}$ is the total number of trials, $\phi_{\mathrm{ijn}}$ is an indicator function that equals 1 if both items $i$ and $j$ are recalled on trial $n$ and equals 0 otherwise, and $d_{k}$ is defined as follows:

$$
\begin{array}{ll}
d_{k}=t_{k+1, n}-t_{k, n} & \text { for } k=\left(1,2,3, \ldots r_{n}-1\right) \\
0 & \text { for } k=r_{n}
\end{array}
$$

where $t_{k, n}$ is equal to the time item $k$ is recalled (or serial position of item $k$ ) on trial $n$ and $r_{n}$ equals the number of items recalled on trial $\mathrm{n}$. Before printing, these raw distances are scaled from 0 to 1 by dividing each $D_{i j}$ by the maximum raw distance. This maximum value is printed along with the distance matrix to allow conversion back to raw form. Since distance measures based upon small recall frequencies (i.e., small values of $\left.\Sigma \phi_{\mathrm{ijn}}\right)$ will not be very stable, an option is provided to allow the cluster analysis to ignore all items with frequencies less than some user-specified minimum.

The clustering algorithm is based upon the procedure suggested by Johnson (1967) with modifications suggested by Gruvaeus and Wainer (1972) to insure that clusters are uniquely ordered on the basis of their similarity with adjacent clusters. Recall interitem distances may be defined in terms of either the total time or the number of items intervening between the recall of each pair of items. These distance measures may be averaged over trials to obtain more stable estimates of subjective distance. The program begins by searching the distance matrix for the pair of items with the greatest similarity (smallest distance). These two items are joined to form the first node in the hierarchy and are subsequently treated as a single unit. For example, in the sample output shown in Figure 1, "peach" and "pear" form the first cluster. The distance between this cluster and all remaining items are recomputed using one of three possible linkage rules: (1) Single Linkage-The distance between the new cluster and some item outside the cluster is set equal to the minimum distance between either item in the cluster and the other item. (2) Average Linkage-The new distance is equal to the average distance between each item in the cluster and some external item. (3) Complete Linkage-The new distance is equal to the maximum distance between items in the cluster and some external item. The distance matrix is then searched for the next most similar pair of items, which are then joined to form the next node in the hierarchy ("saw" and "place" in Figure 1). This process is repeated until all items have been linked together into a single group.

Input. Multiple analyses can be performed in a single run. Input data may be in the form of either a lower triangular distance matrix or raw recall data consisting of a list of words recalled separated by interresponse times. If interresponse times are not available, the constant 1.0 may be used instead; this will yield a distance metric based upon the number of intervening items. These data are used to compute distances between all $n(n-1) / 2$ pairs of recalled items, using average interresponse time or average number of intervening items. Each data set is preceded by a set of cards that specify the options desired, the input and output formats, and subject identification information.

Output. Options are provided to allow printing and/or punching of the interitem distance matrix. Mean distances are printed in the lower half of the matrix and pairwise recall frequencies are printed in the upper half. For each trial, the total number recalled and mean and standard deviation of the trial distance measures are automatically printed. Optionally, the raw input data may also be printed. The results of each cluster analysis 
,

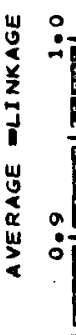

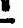

:

1

:

$\overbrace{1}^{1}$

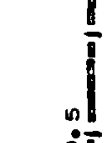

○-

$\frac{1}{0}$
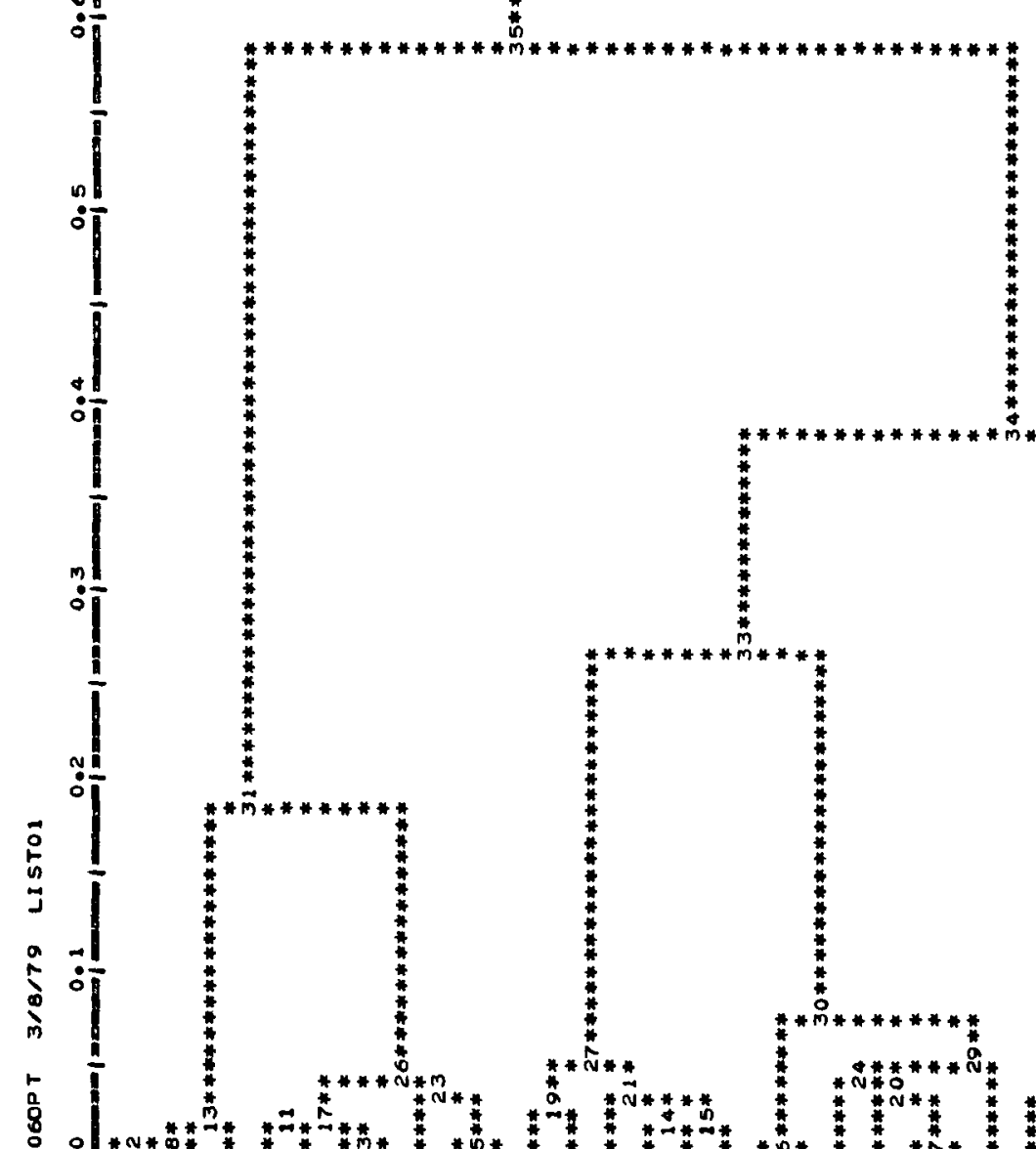
are presented both in the form of a table and in dendographic form (Figure 1). The table lists the elements that form each node of the hierarchy along with the similarity value for that node in both raw and normalized form. The dendograph portrays the same information in graphical form that can be produced on any standard line printer.

Computer and Language. The program is written in FORTRAN IV and was developed on an ITEL AS/6 (IBM 370-compatible) computer. It should run on any system with a FORTRAN IV compiler with little or no modification.

Core and Time Requirements. The program consists of 522 FORTRAN statements plus instructions on comment cards and uses approximately $60 \mathrm{~K}$ bytes of memory, of which $14 \mathrm{~K}$ are used for data storage. A cluster analysis with 35 items requires .6 CPU sec on the ITEL AS/6 or 3.2 CPU sec on an IBM 370-155 II.

Limitations. The program as written will handle up to 50 items total with a maximum of 60 items on any one trial (the trial total may be larger than the overall total because of item repetitions). These limits may be easily modified by redimensioning arrays in the main routine. Because computation time increases rapidly as a function of the total number of items, the practical upper limit on the number of items that can be clustered should be in the range of from 100 to 150 items.

Availability. A program listing with instructions for use may be obtained at no cost by writing to Charles $\mathrm{E}$. Lincoln, Department of Psychology, Rice University,
Houston, Texas 77001. The source deck is also available at a nominal charge to cover postage and duplication costs.

\section{REFERENCE NOTE}

1. Friendly, M. L. Proximity analysis and the structure of organization in free recall (ETS RB-72-3). Princeton, N.J: Educational Testing Service, 1972.

\section{REFERENCES}

Bousfield, W. A. The occurrence of clustering in the recall of randomly arranged associates. Journal of General Psychology, 1953, 49, 229-240.

Bousfield, W. A., \& Sedgewick, C. H. An analysis of sequences of restricted associative responses. Journal of General Psychology, 1944, 30, 149-165.

Friendly, M. L. In search of the M-gram: The structure of organization in free recall. Cognitive Psychology, 1977, 9, 188-249.

Gruvaeus, G. T., \& Wainer, H. C. Some notes on hierarchical clustering procedures. British Journal of Mathematical and Statistical Psychology, 1972, 25, 200-206.

Johnson, S. C. Hierarchical clustering schemes. Psychometrika, 1967, 32, 241-254.

Mille R, G. A. A psychological method to investigate verbal concepts. Journal of Mathematical Psychology, 1969, 6, 169-191.

Sternberg, R. J., \& Tulving, E. The measurement of subjective organization in free recall. Psychological Bulletin, 1977, 84, 539-556.

(Accepted for publication June 3, 1979.) 